\title{
Is Tinnitus Retraining Therapy effective in managing Persistent Tinnitus after Middle Ear Surgery?
}

\author{
Vasu KR ${ }^{1}$, Krishna CV2*, Manideep $\mathrm{C}^{3}$ and Manisha ${ }^{4}$ \\ ${ }^{1}$ Audiologist and Speech Pathologist, Narayana Medical College, India \\ ${ }^{2}$ Department of ENT, Professor, Narayana Medical College and Hospital, India \\ ${ }^{3}$ Resident/ Post Graduate, Narayana Medical College, Nellore, India \\ ${ }^{4}$ Resident/ Post Graduate, Narayana Medical College, Nellore, India
}

Research Article

Volume 4 Issue 1

Received Date: April 18, 2019

Published Date: May 30, 2019

DOI: $10.23880 / 00 A J-16000178$

*Corresponding author: Krishna Chaitanya V, Department of ENT, Professor, Narayana Medical College and Hospital, Andhra Pradesh, India, Tel: 9963337475/ 8008086119; Email: drvkc17@gmail.com

\section{Abstract}

Introduction: Tinnitus, commonly referred to as "ringing in the ears," is phantom auditory perception which can be debilitating condition. Middle ear surgery can give rise to complications like development of Tinnitus in certain patients. This article evaluates patients with persistent Tinnitus managed by Tinnitus Retraining therapy.

Materials and Methods: Prospective study was carried on patients operated for chronic middle ear disease presented with persistent Tinnitus post operatively at completion of 3 months of follow up during January 2016 to December 2017. Level of tinnitus was assessed using Tinnitus Handicap Inventory (THI) scale. These Patients were subjected to Tinnitus Retraining Therapy for 3 months.

Results: Total of 29 patients presented with Persistent Tinnitus Post operatively of which 17 were male and 12 female in age group 21-50 years. Tinnitus was mild (13.79\%), moderate (51.72\%), severe (31.03\%) and Catastrophic (3.44\%) of patients. Frequency, Intensity match and type of noise of each patient for tinnitus retraining therapy was documented where unilateral Tinnitus was seen in 25 and bilateral in 4 patients. Overall improvement in tinnitus observed in 16 $(55.17 \%)$ of patients. Patients with moderate tinnitus showed improvement in Catastrophic and Severe tinnitus. As perception of Tinnitus increased more improvement was observed following therapy and vice versa. Results of Tinnitus Retraining Therapy do not vary based on middle ear surgery performed, laterality of ear and type of noise used to suppress tinnitus.

Discussion: Tinnitus retraining Therapy aims in reducing tinnitus perception by inducing habituation of tinnitus-induced reactions allowing patients to achieve control over tinnitus, live normal life.

Keywords: Tinnitus Retraining Therapy; Chronic Middle Ear Disease 


\section{Otolaryngology Open Access Journal}

Abbreviations: THI: Tinnitus Handicap Inventory; TRT: Tinnitus Retraining Therapy.

\section{Introduction}

Tinnitus, commonly referred to as "ringing in the ears," is a phantom auditory perception which can be annoying or may be debilitating condition [1]. After middle ear surgery expectations of hearing gain in postsurgery period are minimal and can give rise to complications like development of Tinnitus in certain patients. The effective management of tinnitus complaints is often a lengthy and troublesome treatment process involving numerous disciplines.

In persistent tinnitus various treatment modalities have been tried to treat tinnitus which include Pharmacotherapy, Cognitive behavior therapy, Tinnitus masking, Tinnitus retraining therapy and Trans-cranial electric or magnetic stimulation [2]. Tinnitus retraining therapy involves masking of tinnitus at sound perception level in combination with structured counselling sessions [3]. This article provides tools to evaluate patients with persistent Tinnitus following middle Ear Surgery for treatment with Tinnitus Retraining Therapy and to monitor the efficacy of treatment with Tinnitus Retraining therapy.

\section{Materials and Methods}

A prospective study was carried on patients of chronic middle ear diseases and operated, who presented with persistent Unilateral or Bilateral Tinnitus post operatively at the completion of 3 months of follow up in ENT Department from January 2016 to December 2017 were included in the study. Other patients presenting with tinnitus as not meeting the inclusion criteria were excluded from the study. After an informed consent appropriate middle ear surgery was performed in these patients based on their diagnosis which included stapedectomy, tympanoplasty, Myringotomy and Mastoid exploration. Among these patients, group of patients who presented with persistent tinnitus at the completion of 3 months of follow up after the surgery were selected. In these patients the level of tinnitus was assessed using validated Tinnitus Handicap Inventory (THI) [4] scale and the results were documented.

Prior to the commencement of the therapy severity of tinnitus was documented based on patients response over a 25 point questionnaire according to the Tinnitus handicap inventory (THI) scale. Thorough Audiological evaluation of the patient was performed using Pure Tone Audiometry and the frequency match is identified and intensity is matched accordingly. After establishing the grade of tinnitus on Tinnitus handicap inventory (THI) scale score, patients were subjected to Tinnitus Retraining Therapy for a period of 3 months.

After the completion of Tinnitus retraining Therapy (TRT) for three months, Patients were followed up and the results were documented. An improvement in tinnitus severity, Improvement in tinnitus perception, loudness or intensity, Improvement in well-being and disability is measured by Tinnitus handicap inventory (THI) scale and the responses were compared with previous responses before the commencement of Tinnitus Retraining Therapy (TRT).

\section{Results}

A total of 29 patients in our study who presented with Persistent Tinnitus Post operatively owing to the middle ear surgery performed of which 17 were male and 12 female in the age group 21-50 years. Tinnitus retraining therapy was administered in these patients for a period of three months. Prior to administration of the tinnitus retraining therapy the perception of tinnitus in the patients was documented using validated Tinnitus Handicap Inventory (THI) scale as mentioned in the Table 1 below.

\begin{tabular}{|c|c|c|}
\hline Degree of tinnitus & No of patients & Percentage \\
\hline Mild & 4 & 13.79 \\
\hline Moderate & 15 & 51.72 \\
\hline Severe & 9 & 31.03 \\
\hline Catastrophic & 1 & 3.44 \\
\hline
\end{tabular}

Table 1: Tinnitus perception of patients before initiation of tinnitus retraining therapy $(n=29)$.

Patients with persistent Tinnitus at the end of 3 months follow up after middle ear surgery were classified based on the surgical procedure performed and the values were documented in the Table 2 below. 


\section{Otolaryngology Open Access Journal}

\begin{tabular}{|c|c|c|c|}
\hline Surgery & Total No of Surgeries & Patients with tinnitus Before TRT & Improvement (\%) \\
\hline Stapedectomy & 215 & 10 & $6(60.0)$ \\
\hline Mastoidectomy & 139 & 12 & $7(58.33)$ \\
\hline Tympanoplasty & 617 & 5 & $2(40.0)$ \\
\hline Fluid Filled & 78 & 2 & $1(50.0)$ \\
\hline
\end{tabular}

Table 2: Based on Surgery Change in Tinnitus $(n=29)$.

A detailed view of Frequency, Intensity match and type of noise for each patient for tinnitus retraining therapy was documented in the Table 3 mentioned below. From the table it was evident that patients with unilateral Tinnitus were seen in 25 patients. Rest of the 4 patients presented with Bilateral Tinnitus. In the following table Patients presenting with narrow band noise in right ear where Intensity and frequency match was performed based on pure tone audiometry was observed in 11 patients. Improvement in Tinnitus perception was observed in this group in 6(54.54\%) patients. Similarly in left ear with narrow band noise there was tinnitus perception prior to commencement of therapy in 2 patients. Improvement was observed in 1(50.0\%) of patients. Similarly in the following table Patients presenting with white band noise in right ear where Intensity and frequency match was performed based on pure tone audiometry was observed in 6 patients. An improvement was observed in $3(50.0 \%)$ of these patients. Results in left ear with white band noise there was tinnitus perception prior to commencement of therapy in 10 patients. Improvement was observed in 5(50.0\%) of patients. These results also included individualised therapy for each ear in patients presenting with Bilateral Tinnitus also. Detailed work up of these results were documented in Table 3 below.

\begin{tabular}{|c|c|c|c|c|c|}
\hline S. No & EAR & Frequency & Intensity (dB) & Type of tone / noise & Suppression \\
\hline 1 & Rt & $1.5 \mathrm{kHz}$ & 35 & white band & $\mathrm{Y}$ \\
\hline 2 & $\mathrm{Lt}$ & $2 \mathrm{kHz}$ & 50 & narrow band & $\mathrm{Y}$ \\
\hline 3 & Lt & $500 \mathrm{~Hz}$ & 45 & white band & $\mathrm{N}$ \\
\hline 4 & $\mathrm{Rt}$ & $500 \mathrm{~Hz}$ & 30 & narrow band & $\mathrm{Y}$ \\
\hline 5 & Lt & $6 \mathrm{kHz}$ & 40 & white band & $\mathrm{Y}$ \\
\hline 6 & Both & $3 \mathrm{kHz}$ & 75 & narrow band & $\mathrm{N}$ \\
\hline 7 & Lt. & $1 \mathrm{kHz}$ & 50 & white band & $\mathrm{Y}$ \\
\hline 8 & Rt & $2 \mathrm{kHz}$ & 55 & narrow band & $\mathrm{Y}$ \\
\hline 9 & Lt & $2 \mathrm{kHz}$ & 55 & narrow band & $\mathrm{N}$ \\
\hline 10 & Rt & $3 \mathrm{kHz}$ & 60 & white band & $\mathrm{Y}$ \\
\hline 11 & $\mathrm{Lt}$ & $4 \mathrm{kHz}$ & 40 & white band & $\mathrm{N}$ \\
\hline 12 & $\mathrm{Rt}$ & $2 \mathrm{kHz}$ & 55 & narrow band & $\mathrm{Y}$ \\
\hline 13 & $\mathrm{Rt}$ & $3 \mathrm{kHz}$ & 60 & white band & $\mathrm{N}$ \\
\hline 14 & Both & $4 \mathrm{kHz}$ & 40 & white band & $\mathrm{N}$ \\
\hline 15 & Lt & $3 \mathrm{kHz}$ & 60 & white band & $\mathrm{Y}$ \\
\hline 16 & Lt & $4 \mathrm{kHz}$ & 40 & white band & $\mathrm{N}$ \\
\hline 17 & Rt. & $3 \mathrm{kHz}$ & 30 & narrow band & $\mathrm{Y}$ \\
\hline 18 & Both & $1 \mathrm{kHz}$ & 70 & narrow band & $\mathrm{N}$ \\
\hline 19 & $\mathrm{Lt}$ & $750 \mathrm{~Hz}$ & 75 & white band & $\mathrm{N}$ \\
\hline 20 & Rt & $2 \mathrm{kHz}$ & 55 & narrow band & $\mathrm{Y}$ \\
\hline 21 & Both & $2 \mathrm{kHz}$ & 40 & narrow band & $\mathrm{N}$ \\
\hline 22 & $\mathrm{Rt}$ & $3 \mathrm{kHz}$ & 60 & white band & Y \\
\hline 23 & Lt & $4 \mathrm{kHz}$ & 40 & white band & $\mathrm{N}$ \\
\hline 24 & Rt & $2 \mathrm{kHz}$ & 55 & narrow band & Y \\
\hline 25 & $\mathrm{Lt}$ & $3 \mathrm{kHz}$ & 60 & white band & $\mathrm{Y}$ \\
\hline 26 & $\mathrm{Rt}$ & $1 \mathrm{kHz}$ & 55 & narrow band & $\mathrm{N}$ \\
\hline 27 & Lt. & $2 \mathrm{kHz}$ & 35 & white band & $\mathrm{Y}$ \\
\hline 28 & Rt. & $1.5 \mathrm{kHz}$ & 40 & white band & $\mathrm{N}$ \\
\hline 29 & Rt. & $750 \mathrm{~Hz}$ & 35 & narrow band & $\mathrm{N}$ \\
\hline
\end{tabular}

Table 3: Frequency, Intensity match and type of noise for each patient for tinnitus retraining therapy $(n=29)$. 


\section{Otolaryngology Open Access Journal}

From the results of our study we observed an improvement in Tinnitus perception after successful completion of therapy at the end of 3 months. These results were documented in the table IV below.

\begin{tabular}{|c|c|c|}
\hline Perception change in patients & $\mathbf{n = 2 9}$ & Percentage \\
\hline Catastrophic to Catastrophic & 0 & 0 \\
\hline Catastrophic to moderate & 1 & 33.33 \\
\hline Severe to severe & 3 & 11.11 \\
\hline Severe to moderate & 1 & 33.33 \\
\hline Severe to mild & 3 & 22.23 \\
\hline Severe to no tinnitus & 2 & 46.67 \\
\hline Moderate to moderate & 7 & 6.66 \\
\hline Moderate to mild & 1 & 13.33 \\
\hline Moderate to slight & 2 & 33.33 \\
\hline Moderate to no tinnitus & 5 & 75 \\
\hline Mild to mild & 3 & 25 \\
\hline
\end{tabular}

Table 4: Tinnitus perception after tinnitus retraining therapy.

\section{Discussion}

Tinnitus is common in patients with chronic middle ear diseases. It is usual that Tinnitus in the setting of otosclerosis may show more consistent improvement after successful stapedectomy surgery [5]. Relief from tinnitus after successful stapedectomy may be due to an auditory effect whereby increased conductive hearing input drowns out spontaneous output from the cochlear nerve or nuclei. The mechanism of onset of tinnitus as a complication of chronic otitis media is unknown but recovery from tinnitus attributable to the conductive system of the middle ear may be expected after tympanoplasty [6]. However, after tympanoplasty, recovery from tinnitus attributable to the conductive system of the middle ear may be expected.

In general it is observed that postoperative Audiological gain is an important factor determining outcome of tinnitus in these types of otological surgeries [7]. In the present study the results show an overall improvement in tinnitus perception in $16(55.17 \%)$ of patients owing to a positive result in these patients following Tinnitus retraining therapy. In general it is believed that retraining counselling acts to decrease the level of stimulation from the cortical areas of the brain to the limbic and autonomic nervous systems and to decrease the general level of activity within these two systems [8]. Tinnitus retraining therapy appears to be highly effective used to treat all types of patients, does not require frequent visits, and does not interfere with hearing, and there are no negative side effects.
When the improvement in severity perception of tinnitus was observed, the group of patients presenting with moderate tinnitus had shown overall improvement in Catastrophic and Severe tinnitus. We also observed that as the perception of Tinnitus increased more improvement was observed following therapy and vice versa. Also from our study we infer that the results of Tinnitus Retraining Therapy do not vary based on middle ear surgery performed, laterality of the ear and the type of noise used to suppress tinnitus. As we have clearly stated in our previous studies Tinnitus retraining Therapy aims in reducing the tinnitus perception by inducing habituation of tinnitus-induced reactions allowing patients to achieve control over their tinnitus, live a normal life, and participate in everyday activities [9].

\section{Conclusion}

Tinnitus Retraining Therapy we believe when implemented properly is effective in treating tinnitus. However it does not promise a permanent cure with high success rate but it is justifiable to promote it as an effective treatment for tinnitus patients owing to its minimal side effects.

\section{References}

1. Langguth B (2011) A review of tinnitus symptoms beyond 'ringing in the ears': a call to action. Curr Med Res Opin 27(8): 1635-1643.

2. Maggon NV, Sethi A, Gupta A (2017) Noise Induced Hearing Loss with Tinnitus: Does TRT Help? Bengal 


\section{Otolaryngology Open Access Journal}

Journal of Otolaryngology and Head Neck Surgery 25(1): 39-45.

3. Cima RF, Maes IH, Joore MA, Scheyen DJ, El Refaie A, et al. (2012) Specialised treatment based on cognitive behaviour therapy versus usual care for tinnitus: a randomised controlled trial. Lancet 379(9830): 19511959.

4. Newman CW, Jacobson GP, Spitzer JB (1996) Development of the tinnitus handicap inventory. Arch Otolaryngol Head Neck Surg 122(2): 143-148.

5. Van Rompaey V, Yung M, Claes J, Häusler R, Martin C, et al. (2009) Prospective effectiveness of stapes surgery for otosclerosis in a multicenter audit setting: feasibility of the Common Otology Database as a benchmark database. Otol Neurotol 30(8): 11011110.
6. Baba S, Yagi T, Fujikura $T$ (2004) Subjective evaluation and overall satisfaction after tympanoplasty for chronic simple suppurative otitis media. J Nippon Med Sch 71(1): 17-24.

7. Habesoglu M, Habesoglu TE, Karatas C, Tosun A, Gursel AO, et al. (2013) Is there any predictor for tinnitus outcome in different types of otologic surgery?. Eur Arch Otorhinolaryngol 270(8): 22252229.

8. Jastreboff PJ, Jastreboff MM (2000) Tinnitus retraining therapy (TRT) as a method for treatment of tinnitus and hyperacusis patients. J Am Acad Audiol 11(3): 162-177.

9. Reddy KV, Chaitanya VK, Babu GR (2019) Efficacy of Tinnitus Retraining Therapy, A Modish Management of Tinnitus: Our Experience. Indian J Otolaryngol Head Neck Surg 71(1): 95-98. 